\title{
Cortical multisensory integration-a special role of the agranular insular cortex?
}

\author{
Daniela Brunert ${ }^{1} \cdot$ Markus Rothermel $^{1}$ [D
}

Received: 28 April 2020 / Revised: 4 May 2020 / Accepted: 7 May 2020 / Published online: 26 May 2020

(C) The Author(s) 2020

Multisensory integration describes the act of combining sensory information from different sources to form a cohesive real-world picture. It is considered one of the fundamental functions of the brain [3], but its exact mechanisms still pose an unresolved question in modern neuroscience. No matter if we watch TV, cross a road, eat lunch, or spend time with a beloved person, we experience most of our activities with more than one sensory modality, demonstrating the everyday importance of multisensory integration. How much we rely on its function is especially noticeable in instances when a multisensory mismatch creates problems e.g. if the audio feed in a video call is delayed or inconsistent signals between visual and vestibular input systems create motion sickness (e.g. virtual reality cyber-sickness).

One of the most complex examples of multisensory integration is flavor perception. Flavor not only involves the combination of gustatory, olfactory, and trigeminal inputs but also visual, auditory, and somatosensory stimuli contribute to flavor perception [10]. Where these modalities, especially gustation and olfaction, are combined to form the perception of a certain flavor is still not fully understood. Multiple sites have been implicated so far including the insula, operculum, caudal orbitofrontal cortex (OFC), and anterior cingulate cortex, theorized to form a kind of "flavor network" [6]. It is currently under debate if the OFC is the first area capable of responding to both olfactory and gustatory stimuli or if already primary olfactory and gustatory cortices themselves participate in the processing of multimodal chemosensory information [5].

In this issue of the Pflügers Archiv - European Journal of Physiology, Mizoguchi et al. present novel data on how the insular cortex, including the primary taste cortex, could be

This article is a commentary to the original article https://doi.org/10.1007/ s00424-020-02399-w

Markus Rothermel

m.rothermel@sensorik.rwth-aachen.de

1 Department of Chemosensation, AG Neuromodulation, Institute for Biology II, RWTH Aachen University, 52074 Aachen, Germany involved in the integration of olfactory and gustatory cues. The authors used voltage-sensitive dye (VSD) imaging to examine spatiotemporal dynamics in taste and olfactory cortical areas following chorda tympani (CT) nerve and olfactory bulb (OB) stimulation in the anesthetized rat. This is possible by using a preparational approach that allows the authors to visualize large parts of the cerebral cortex around the rhinal fissure and middle cerebral artery. This includes the insular and piriform cortex, the primary olfactory cortex. The authors recorded optical signals in parallel from these areas while stimulating the chorda tympani nerve or the olfactory bulb electrically in order to precisely control input timing and increase reliability. Chorda tympani stimulation elicited the largest VSD signals in the rostral dysgranular insular cortex $(\mathrm{rDI})$ with smaller albeit significant signals detectable in the secondary somatosensory area/insular oral region but no signals in the piriform cortex. OB stimulation elicited the largest signals in the anterior and posterior piriform cortex but no signals in gustatory areas. Simultaneous stimulation did not change signals in gustatory or olfactory areas compared with stimulation of $\mathrm{CT}$ or $\mathrm{OB}$ alone except in the rostral agranular insular cortex (rAI). Here, unimodal signals seemed to be ineffective but simultaneous stimulation additively increased the measured signal amplitude. Based on these findings, the authors conclude that rather than in the primary sensory cortices, multisensory integration primarily happens in the rAI which is seen as part of the orbitofrontal cortex [4]. Thus, they confirm the findings of several human fMRI studies that highlighted the ventral insula as one of the areas that exhibit additive or even super additive effects to bimodal sensory presentation [1,7-9]. Different publications have, however, shown gustatory responses in the piriform cortex [2] as well as olfactory responses in the gustatory cortex [5]. More studies are required to clarify how far orbitofrontal multisensory integration is distinct from multisensory responses in primary sensory and other lower brain areas.

In summary, Mizoguchi et al. contribute to elucidating the question of how the multimodal sense of "flavor" is processed, something essential if we want to understand the 
complex interplay of the factors controlling phenomena like cravings and hedonic value of food and ultimately, dietary choices, and the amount of food intake. It furthermore highlights the potential of optical imaging for the elucidation of multisensory integration giving a fast overview of cortical signals over relatively large areas.

Acknowledgements Open Access funding provided by Projekt DEAL.

Funding information This work was funded by the Deutsche Forschungsgemeinschaft (DFG, German Research Foundation; RO4046/2-1 and /2-2, Emmy Noether Program and the Research Training Group 2416 "MultiSenses - MultiScales: Novel approaches to decipher neural processing in multisensory integration" 368482240/ GRK2416) and by a grant from the Interdisciplinary Centre for Clinical Research within the faculty of Medicine at the RWTH Aachen University (IZKF TN1-7 532007).

Open Access This article is licensed under a Creative Commons Attribution 4.0 International License, which permits use, sharing, adaptation, distribution and reproduction in any medium or format, as long as you give appropriate credit to the original author(s) and the source, provide a link to the Creative Commons licence, and indicate if changes were made. The images or other third party material in this article are included in the article's Creative Commons licence, unless indicated otherwise in a credit line to the material. If material is not included in the article's Creative Commons licence and your intended use is not permitted by statutory regulation or exceeds the permitted use, you will need to obtain permission directly from the copyright holder. To view a copy of this licence, visit http://creativecommons.org/licenses/by/4.0/.

\section{References}

1. de Araujo IE, Rolls ET, Kringelbach ML, McGlone F, Phillips N (2003) Taste-olfactory convergence, and the representation of the pleasantness of flavour, in the human brain. Eur J Neurosci 18: 2059-2068

2. Maier JX, Blankenship ML, Li JX, Katz DB (2015) A Multisensory Network for Olfactory Processing. Curr Biol 25:2642-2650

3. Pennartz CMA (2015) The brain's representational power: on consciousness and the integration of modalities. The MIT Press, Cambridge

4. Rempel-Clower NL (2007) Role of orbitofrontal cortex connections in emotion. Ann N Y Acad Sci 1121:72-86

5. Samuelsen CL, Fontanini A (2017) Processing of Intraoral Olfactory and Gustatory Signals in the Gustatory Cortex of Awake Rats. J Neurosci 37:244-257

6. Small DM, Prescott J (2005) Odor/taste integration and the perception of flavor. Exp Brain Res 166:345-357

7. Small DM, Jones-Gotman M, Zatorre RJ, Petrides M, Evans AC (1997a) Flavor processing: more than the sum of its parts. Neuroreport 8:3913-3917

8. Small DM, Jones-Gotman M, Zatorre RJ, Petrides M, Evans AC (1997b) A role for the right anterior temporal lobe in taste quality recognition. J Neurosci 17:5136-5142

9. Small DM, Voss J, Mak YE, Simmons KB, Parrish T, Gitelman D (2004) Experience-dependent neural integration of taste and smell in the human brain. J Neurophysiol 92:1892-1903

10. Spence C (2015) Multisensory flavor perception. Cell 161:24-35

Publisher's note Springer Nature remains neutral with regard to jurisdictional claims in published maps and institutional affiliations. 